\title{
Oscillation Conditions for a Type of Second Order Neutral Differential Equations with Impulses
}

\author{
Ubon Akpan Abasiekwere*, Edwin Frank Nsien, Imoh Udo Moffat \\ Department of Mathematics and Statistics, Faculty of Science, University of Uyo, Uyo, Nigeria
}

Email address:

ubeeservices@yahoo.com (U. A. Abasiekwere), ed_nsien@yahoo.com (E. F. Nsien), moffitto@yahoo.com (I. U. Moffat)

*Corresponding author

\section{To cite this article:}

Ubon Akpan Abasiekwere, Edwin Frank Nsien, Imoh Udo Moffat. Oscillation Conditions for a Type of Second Order Neutral Differential Equations with Impulses. American Journal of Applied Mathematics. Vol. 5, No. 4, 2017, pp. 119-123. doi: 10.11648/j.ajam.20170504.14

Received: October 20, 2016; Accepted: November 14, 2016; Published: August 24, 2017

\begin{abstract}
In this paper, we study a certain type of second order linear neutral differential equation with constant impulsive jumps. This type of equation is known always to possess an unbounded non-oscillatory solution. The method and technique of impulse imposition used here is due to studies by Bainov and Simeonov [1]. By assuming, amongst other conditions, that the constant coefficient of the equation in question lies between zero and one and the delay function is non-decreasing, it is shown that all bounded solutions of the said neutral impulsive equation are oscillatory.
\end{abstract}

Keywords: Bounded, Oscillations, Second-order, Neutral, Delay, Impulsive, Differential Equation

\section{Introduction}

In this study, we consider a type of second order linear neutral differential equation of the form

$$
\left\{\begin{array}{r}
{[y(t)-p y(t-\tau)]^{\prime \prime}=q(t) y(g(t)),} \\
t \neq t_{k} \\
\Delta\left[y\left(t_{k}\right)-p y\left(t_{k}-\tau\right)\right]^{\prime}=q_{k} y\left(g\left(t_{k}\right)\right), \\
\forall t=t_{k},
\end{array}\right.
$$

with constant impulsive jumps, where $\tau>0$, $0 \leq t_{0}<t_{1}<\cdots<t_{k}<\cdots$ with $\lim _{k \rightarrow+\infty} t_{k}=+\infty$, $\Delta y^{(i)}\left(t_{k}\right)=y^{(i)}\left(t_{k}^{+}\right)-y^{(i)}\left(t_{k}^{-}\right), i=0,1, k \in N$, and $y\left(t_{k}^{-}\right), y\left(t_{k}^{+}\right)$represent the left and right limits of $y(t)$ at $t=t_{k}$, respectively. Our aim here is to establish some criteria that guarantee the oscillation of all its bounded solutions.

The development of the theory of impulsive differential equations is one of the recent trends in the history of qualitative theory of differential equations [14-28]. There are many monographs related to this subject [1, 29-31], etc. In this direction, credit must be given to Professor Drumi Bainov, Lakshmikantham and Pavel Simeonov, to mention just a few, for their contributions in the development of the oscillatory and non-oscillatory properties for various classes of impulsive differential equations with delay and with advanced arguments.

Furthermore, the theory of oscillations of neutral impulsive differential equations is gradually occupying a central place among the theories of oscillations of impulsive differential equations. This could be due to the fact that neutral impulsive differential equations play fundamental roles in the present drive to further develop information technology. Indeed, neutral differential equations appear in networks containing lossless transmission lines (as in high-speed computers where the lossless transmission lines are used to interconnect switching circuits) [8]. They also appear in problems dealing with vibrating masses attached to an elastic as well as the Euler equation in some vibrational problems [2, 3, 5-7]. Very recently, the unending list of results on oscillation and nonoscillation of solutions of first order neutral delay impulsive differential equations has increased [8, 9, 11-13].

However, we notice that oscillation theory of second order neutral differential equations with impulses is still at its infancy. The dearth of results in this area is seen in the following articles [32-35, 37-38]. Cheng and Chu [32] established some necessary and sufficient conditions for the oscillation of a second-order linear neutral impulsive differential equation with advanced arguments and constant coefficients of the form 


$$
\left\{\begin{array}{l}
{[x(t)-p x(t-\tau)]^{\prime \prime}=q(t-\sigma), t \neq t_{k}} \\
\Delta\left[x\left(t_{k}\right)-p x\left(t_{k}-\tau\right)\right]^{\prime}=q x\left(t_{k}-\sigma\right)
\end{array}\right.
$$

where $q, q_{1}>0, \quad p, \tau, \sigma<0$ and $\Delta x^{(i)}\left(t_{k}\right)=x^{(i)}\left(t_{k}^{+}\right)-$ $x^{(i)}\left(t_{k}^{-}\right), \mathrm{i}=0,1, k \in N$. Tripathy and Santra [35] obtained sufficient conditions for oscillation of all solutions of a class of nonlinear neutral impulsive differential equations of second order with deviating argument and variable coefficients of the form

$$
\left\{\begin{array}{l}
\left(r(t)(\mathrm{x}(t)+p(t) \mathrm{x}(t-\tau))^{\prime}\right)^{\prime}+q(t) G(\mathrm{x}(t-\sigma))=0, t \neq t_{k} \\
\Delta\left(r\left(t_{k}\right)\left(\mathrm{x}\left(t_{k}\right)+p_{k} \mathrm{x}\left(t_{k}-\tau\right)\right)^{\prime}\right)+q_{k} G\left(\mathrm{x}\left(t_{k}-\sigma\right)\right)=0,
\end{array}\right.
$$

where $\tau, \sigma \in(0,+\infty), p_{k}, r_{k}$ and $q_{k}$ are constants $k \in N$. Their result became a generalization of that obtained by Bainov and Dimitrova [36]. Bonotto et al. [33] investigated the oscillation of solutions of a certain type of second-order neutral delay differential systems

$$
\left\{\begin{array}{l}
\left(r(t)(\mathrm{x}(t)+p(t) \mathrm{x}(t-\tau))^{\prime}\right)^{\prime}+f(t, y(t), \mathrm{x}(t-\delta))=0, t \neq t_{k} \\
\mathrm{x}\left(t_{k}\right)=I_{k}\left(\mathrm{x}\left(t_{k}^{-}\right)\right), x^{\prime}\left(t_{k}\right)=J_{k}\left(\mathrm{x}^{\prime}\left(t_{k}^{-}\right)\right), k=1,2,3, \cdots \\
\mathrm{x}(t)=\varphi(t), t_{0}-\sigma \leq t \leq t_{0},
\end{array}\right.
$$

where $p(t) \in P C^{1}\left(\left[t_{0},+\infty\right), R\right), r(t)$ is a positive continuous function defined on $\left[t_{0},+\infty\right), \delta, \tau \in(0,+\infty), \sigma=\max \{\delta, \tau\}$ and $\varphi, \varphi^{\prime}:\left[t_{0}-\sigma, t_{0}\right] \rightarrow R$. They established adequate impulse controls under which the system remained oscillatory after undergoing controlled abrupt perturbations (called impulses). Abasiekwere and Moffat [37] examined the oscillations of a class of second order linear neutral impulsive ordinary differential equations with variable coefficients and constant retarded arguments and obtained sufficient conditions ensuring the oscillation of all solutions. Again, Abasiekwere, et al [38] closely considered a certain type of second order delay differential equations with constant impulsive jumps and obtained sufficient conditions for the oscillation of all its bounded solutions.

Here, in this paper, we are concerned with oscillations of all bounded solutions of a type of second order linear neutral differential equation with constant impulsive jumps.

The theory of differential equations in general is based on the behavior of processes under the influence of short-time perturbations. The duration of these perturbations are extremely small and can be ignored compared to the total duration of the process itself. Therefore, they are regarded as 'momentary', that is, the perturbations are of impulsive type.

In ordinary differential equations, the solutions are continuously differentiable sometimes at least once, whereas the impulsive differential equations generally possess noncontinuous solutions. Since the continuity properties of the solutions play an important role in the analysis of the behaviour, the techniques used to handle the solutions of impulsive differential equations are fundamentally different including the definitions of some of the basic terms. In this section, we examine some of these changes [11].

In effect, the solution $y(t)$ for $t \in\left[t_{0}, T\right)$ of a given impulsive differential equation or its first derivative $y^{\prime}(t)$ is a piece-wise continuous function with points of discontinuity $t_{k} \in\left[t_{0}, T\right), t_{k} \neq t, 0 \leq k<\infty$. Consequently, in order to simplify the statements of the assertions later, we introduce the set of functions $P C$ and $P C^{r}$ which are defined as follows:

Let $r \in N, D:=[T, \infty) \subset R$ and let $\mathrm{S}:=\left\{t_{k}\right\}_{k \in N}$ be fixed. Except stated otherwise, we will assume that the elements of $S$ are moments of impulsive effects and satisfy the property:

C 1.1: $0<t_{1}<t_{2}<\cdots$ and $\lim _{k \rightarrow \infty} t_{k}=+\infty$.

We denote by $P C(\mathrm{D}, \mathrm{R})$ the set of all values $\varphi: D \rightarrow R$ which is continuous for all $t \in D, t \notin S$. They are continuous from the left and have discontinuity of the first kind at the points for $t \in S$. By $P C^{r}(D, R)$, we denote the set of functions $\varphi: D \rightarrow R$ having derivative $\frac{d^{j} \varphi}{d t^{j}} \in P C(D, R), 0 \leq j \leq r[1,4]$. To specify the points of discontinuity of functions belonging to $P C$ and $P C^{r}$, we shall sometimes use the symbols $P C(D, R ; S)$ and $P C^{r}(D, R ; S), r \in N[8,11,12]$.

Without further mentioning, we will assume throughout this paper that every solution $y(t)$ of equation (1) that is under consideration here, is continuous to the right and is nontrivial. That is, $y(t)$ is defined on some half-line $\left[T_{y}, \infty\right)$ and $\sup \{|y(t)|: t \geq T\}>0$ for all $T \geq T_{y}$. This solution is said to be a regular solution and we assume that our neutral impulsive differential equation possesses this type of solution.

Let $(P)$ be some property of the solution of $y(t)$ of an impulsive differential equation, which can be fulfilled for some $t \in R$. Hereafter, we shall say that the function $y(t)$ enjoys the property $(P)$ finally, if there exists $T \in R$ such that $y(t)$ enjoys the property $(P)$ for all $t \geq T[1]$.

Definition 1.1 The solution $y(t)$ of an impulsive differential equation is said to be

i) finally positive (finally negative) if there exist $T \geq 0$ such that $y(t)$ is defined and is strictly positive (negative) for $t \geq T$ [9];

ii) non-oscillatory, if it is either finally positive or finally negative; and

iii) oscillatory, if it is neither finally positive nor finally negative $[1,8]$.

Remark 1.1: All functional inequalities considered in this paper are assumed to hold finally, that is, they are satisfied for all $t$ large enough.

Remark 1.2: Without loss of generality we will deal only with the positive solutions of equation (1).

\section{Statement of the Problem}

At this point, may we recall that the problem under 
consideration is the second order linear neutral impulsive differential equation of the form

$$
\left\{\begin{aligned}
& {[y(t)-p y(t-\tau)]^{\prime \prime} }=q(t) y(g(t)), \\
& t \geq t_{0}, t \notin S \\
& \Delta\left[y\left(t_{k}\right)-p y\left(t_{k}-\tau\right)\right]^{\prime}=q_{k} y\left(g\left(t_{k}\right)\right), \\
& t_{k} \geq t_{0}, \forall t_{k} \in S,
\end{aligned}\right.
$$

where $p \in(0,1) \quad, \quad q_{k} \geq 0, \quad q(t) \in P C\left(\left[t_{0}, \infty\right), R_{+}\right)$, $g(t) \in C\left(\left[t_{0}, \infty\right), R\right), \lim _{t \rightarrow \infty} g(t)=\infty, \tau>0$. The second order neutral delay differential equation (2) is a system consisting of a differential equation together with an impulsive condition in which the second order derivative of the unknown function appears in the equation both with and without delay. This type of equation (2) is known to always admit an unbounded non-oscillatory solution. It is worth mentioning here that our aim is not to find the unknown function or solution $y(t)$, but to determine its nature and behavior in oscillatory sense. We, therefore, seek to establish conditions for which all bounded solutions of equation (2) are oscillatory.

\section{Main Results}

The following theorem is an extension of Theorem 4.6.1 found on page 255 as identified in the monograph by Erbe et al [10]. The technique and method used here is due to studies by Bainov and Simeonov [1].

Theorem 3.1: Assume that

i) $0<p<1, \quad \tau>0$ are constants, $q_{k} \geq 0$;

ii) $g(t) \leq t$ and $g(t)$ is non-decreasing for $t \geq t_{0}$;

$$
\text { iii) } \begin{aligned}
\limsup _{t \rightarrow \infty} & {\left[\int_{g(t)}^{t}(s-g(t)) q(s) d s+\right.} \\
& \left.+\sum_{g(t) \leq t_{k}<t}\left(t_{k}-g(t)\right) q_{k}\right]>1 .
\end{aligned}
$$

Then every bounded solution $y(t)$ of equation (2) is oscillatory.

Proof: Assume by contradiction that $y(t)$ is a finally positive bounded solution of equation (2). Define

$$
z(t)=y(t)-p y(t-\tau) .
$$

We have $z^{\prime \prime}(t)>0$ for $t \geq T \geq t_{0}, \Delta z^{\prime}\left(t_{k}\right)>0$ for $k: t_{k} \geq T \geq t_{0}$. If $z^{\prime}(t), \Delta z\left(t_{k}\right)>0$ for $t \geq T^{\prime}>T$ and $k: t_{k} \geq T^{\prime}>T$, then $\lim _{t \rightarrow \infty} z(t)=\infty$, which contradicts the boundedness of $y(t)$. Therefore, $z^{\prime}(t), \Delta z\left(t_{k}\right) \leq 0$ for $t \geq T$ and $k: t_{k} \geq T$.

Here, we observe that there exists two possibilities for $z(t)$ : i) $z(t)>0$ for $t \geq T$;

ii) $z(t)<0$ for $t \geq T^{\prime \prime} \geq T$.

In case (a), we integrate equation (2) from $s$ to $t$ and obtain

$$
z^{\prime}(t)-z^{\prime}(s)=\int_{s}^{t} q(s) y(g(u)) d u++\sum_{s \leq t_{k} \leq t} q_{k} y\left(g\left(t_{k}\right)\right) .
$$

Again, integrating equation (5) in $s$ from $g(t)$ to $t$, we obtain

$$
\begin{aligned}
& z^{\prime}(t)(t-g(t))-z(t)+z(g(t))=\int_{g(t)}^{t} \int_{s}^{t} q(u) y(g(u)) d u d s+ \\
& \quad+\sum_{g(t) \leq t_{k} \leq t} \sum_{s \leq t_{k} \leq t} q_{k} y\left(g\left(t_{k}\right)\right) \\
& =\int_{g(t)}^{t}(s-g(t)) q(s) y(g(s)) d s+\sum_{g(t) \leq t_{k} \leq t}\left(t_{k}-g(t)\right) \times q_{k} y\left(g\left(t_{k}\right)\right) \\
& >\int_{g(t)}^{t}(s-g(t)) q(s) z(g(s)) d s+\sum_{g(t) \leq t_{k} \leq t}\left(t_{k}-g(t)\right) \times q_{k} z\left(g\left(t_{k}\right)\right) \\
& \geq z(g(t))\left[\int_{g(t)}^{t}(s-g(t)) q(s) d s+\sum_{g(t) \leq t_{k} \leq t}\left(t_{k}-g(t)\right) q_{k}\right]
\end{aligned}
$$

Hence for $t \geq T$,

$$
z(t)+z(g(t))\left[\int_{g(t)}^{t}(s-g(t)) q(s) d s+\sum_{g(t) \leq t_{k} \leq t}\left(t_{k}-g(t)\right) q_{k}-1\right] \leq 0
$$

which contradicts the positivity of $z(t)$ and condition (3).

In case (b), we have that

$$
y(t)<p y(t-\tau)<p^{2} y(t-2 \tau)<\cdots<p^{n} y(t-n \tau)
$$

for $t \geq T_{2}+n \tau$, which implies that $\lim _{t \rightarrow \infty} y(t)=0$. Consequently $\lim _{t \rightarrow \infty} z(t)=0$. This is a contradiction and therefore completes the proof of Theorem 3.1.

Remark 3.1: It can be shown that Theorem 3.1 is also true when the coefficient $p=0$.

\section{Conclusion}

In this paper, we are mainly concerned with oscillating systems which remain oscillating after being perturbed by instantaneous changes of state or impulsive constant jumps. We considered a certain type of second-order neutral delay differential system and provided sufficient conditions governing the impulse operators acting on the system so that its bounded solutions are oscillatory. Here, we are able to demonstrate how well-known mathematical techniques and methods, after suitable modification, is extended in proving an oscillatory theorem for a class of second order neutral impulsive differential equations (1). 


\section{References}

[1] D. D. Bainov and P. S. Simeonov, Oscillation Theory of Impulsive Differential Equations, International Publications Orlando, Florida, 1998.

[2] R. D. Driver, A mixed neutral system, Nonlinear Anal. 8, 1984, pp. 155-158.

[3] G. S. Ladde, V. Lakshmikantham and B. G. Zhang, Oscillation Theory of Differential Equations with Deviating Arguments, Marcel Dekker, New York, 1987.

[4] V. Lakshmikantham, D. D. Bainov and P. S. Simeonov, Theory of Impulsive Differential Equations, World Scientific, Singapore, 1989.

[5] W. T. Li, Interval oscillation criteria of second-order halflinear functional differential equations, Appl. Math. Comput. 155, 2004, pp. 451-468.

[6] X. Lin, Oscillation of second-order nonlinear neutral differential equations, J. Math. Anal. Appl. 309, 2005, pp. 442-452.

[7] F. Meng and J. Wang, Oscillation criteria for second order quasilinear neutral delay differential equations, J. Indones. Math. Soc. (MIHMI) 10, 2004, pp. 61-75.

[8] I. O. Isaac and Z. Lipcsey, Oscillations of Scalar Neutral Impulsive Differential Equations of the First Order with variable Coefficients, Dynamic Systems and Applications, 19, 2010, pp. 45-62.

[9] I. O. Isaac, Z. Lipcsey and U. J. Ibok, Nonoscillatory and Oscillatory Criteria for First Order Nonlinear Neutral Impulsive Differential Equations, Journal of Mathematics Research; Vol. 3 Issue 2, 2011, pp. 52-65.

[10] L. H. Erbe, Q. Kong and B. G. Zhang, Oscillation Theory for Functional Differential Equations, Dekker, New York, 1995.

[11] I. O. Isaac and Z. Lipcsey, Oscillations in Linear Neutral Delay Impulsive Differential Equations with Constant Coefficients, Communications in Applied Analysis, 14, 2010, pp. 123-136.

[12] I. O. Isaac and Z. Lipcsey, Linearized Oscillations in Nonlinear Neutral Delay Impulsive Differential Equations, Journal of Modern Mathematics and Statistics, 3, 2009, pp. $17-21$.

[13] I. O. Isaac and Z. Lipcsey, Oscillatory Conditions on Both Directions for a Nonlinear Impulsive Differential Equation with Deviating Arguments, Journal of Mathematics Research, 3, 2011, pp. 48-51.

[14] D. D. Bainov, M. B. Dimitrova and A. B. Dishliev, Oscillationof the Bounded Solutions of Impulsive Differential-Difference Equations of Second Order, Applied Mathematics and Computation, 114, 2000, pp. 61-68.

[15] D. D. Bainov and P. S. Simeonov, The Second Method of Lyapunov for Systems with an Impulse Effect, Tamkang J. Math. 16, 1985, pp. 19-40.

[16] D. D. Bainov and P. S. Simeonov, Stability with respect to Part of the Variables in Systema with Impulsive Effect, Journal of Mathematical Analysis and Applications, 1986, pp. 247-263.

[17] C. Yong-Shao and F. Wei-Zhen, Oscillation of Second Order Nonlinear Ordinary Differential Equations with Impulses,
Journal of Mathematical Analysis and Applications, 210, 1997, pp. 150-169.

[18] A. B. Dishliev and D. D. Bainov, Dependence upon Initial Conditions and Parameter of Impulsive Differential Equations with Variable Structure, International Journal of Theoretical Physics, 29 No. 6, 1990, pp. 655-675.

[19] S. I. Gurgula, Investigation of the Stability of Solutions of Impulse Systems by Lyapunov's Second Method, Ukrainian Math., 1, 1982, pp. 400-103.

[20] S. V. Krishna, J. Vasundlara Devi and K. Satyavani, Boundedness and Dichotomies for Impulse Equations, Journal of Mathematical Analysis and Applications, 158, 1991, pp. 352-375.

[21] G. K. Kulev and D. D. Bainov, Lipschitz Stability of Impulsive Systems of Differential Equations, International Journal of Theoretical Physics 30, 1991, pp. 737-756.

[22] V. Lakshmikantham, D. D. Bainov and P. S. Simeonov, Theory of Impulsive Differential Equations, World Scientific Publishing Company Ltd, Singapore, 1989.

[23] V. Lakshmikantham and X. Liu, On Quasistability for Impulsive Differential Systems, Nonlinear Analysis, 13, No. 7, 1989, pp. 819-828.

[24] M. Peng and Ge, Oscillation Criteria for Second Order Nonlinear Differential Equations with Impulses, Computers and Mathematics with Applications, 30, 2000, pp. 217-225.

[25] A. M. Samoilenko and N. A. Perestyuk, Stability of the Solutions of Differential Equations with Impulse Effect, Differential Equations, 11, 1977, pp. 1981-1992.

[26] P. P. Zabreiko, D. D. Bainov and S. I. Kostadinov, Characteristic Exponents of Impulsive Differential Equations in a Banach Space, International Journal of Theoretical Physics, 27, 1988, pp. 721-743.

[27] Y. Zhang, A. Zhao and J. Yan, Oscillation Criteria for Impulsive Differential Equations, Journal of Mathematical Analysis and Applications, 205, 1997, pp. 461-470.

[28] I. O. Isaac, Z. Lipcsey and U. J. Ibok, Linearized Oscillations in Autonomous Delay Impulsive Differential Equations, British Journal of Mathematics \& Computer Science, 4(21): 2014, pp. 3068-3076.

[29] A. M. Samoilenko and N. A. Perestyuk, Impulsive differential Equations, World Scientific Publishing Company Ltd, Singapore, 1995.

[30] R. P. Agarwal, M. Benchohra, D. O'Regan and A. Ouahab, Second order Impulsive dynamic equations on time scales, Functional differential equations II, No. 3-4, 2004, pp. 223-234.

[31] S. G. Deo and S. G. Pandit, Differential systems involving impulses, Lecture Notes 954, Springer-Verlag, Berlin. differential equations Arch. Mat. 41, 1982, pp. 352-362.

[32] J. Cheng and Y. Chu, Oscillations of Second-Order Neutral Impulsive Differential Equations, Journal of Inequalities and Applications; doi: 10.1155/2010/493927: 1-29.

[33] E. M. Bonotto, L. P. Gimenes and M. Federson, Oscillation for a second-order neutral differential equation with impulses, Cadernos De Matematica; 09: 2008, pp. 169-190.

[34] Y. Sun, Oscillation of Certain Second-Order Sub-Half-Linear Neutral Impulsive Differential Equations, Discrete Dynamics in Nature and Society; doi: 10.1155/2011/195619: 2011, pp. 110 . 
[35] A. Tripathy and S. Santra, Oscillation Properties of a Class of Second Order Impulsive Differential Systems of Neutral Type, Functional Differential Equations; 23(1-2): 2016, pp. 57-71.

[36] D. D. Bainov and M. B. Dimitrova, Oscillation of sub and super linear impulsive differential equations with constant delay, Applicable Analysis; 64, 1977, pp. 57-67.

[37] U. A. Abasiekwere and I. U. Moffat, Oscillation Theorems for Linear Neutral Impulsive Differential Equations of the Second
Order with Variable Coefficients and Constant Retarded Arguments, Applied Mathematics, Vol. 7 No. 3, 2017, pp. 3943. doi: $10.5923 / \mathrm{j} . \mathrm{am} .20170703 .01$.

[38] U. A. Abasiekwere, E. F. Nsien and I. U. Moffat, On the Existence of Bounded Oscillatory Solutions of Impulsive Delay Differential Equations of the Second Order, International Journal of Mathematics Trends and Technology, 2017, 48(2), pp. 6-10, ISSN: 2231-5373. 